DOI: $10.12957 /$ demetra.2015.16759

\title{
Distribuição dos valores de resistência, reactância e ângulo de fase entre indígenas escolares Kaingáng, Rio Grande do Sul, Brasil
}

\section{Distribution of resistence, reactance and phase angle values among indigenous students Kaingáng, Rio Grande do Sul, Brazil}

Nicole Lovise Gonzaga Oliveira Santos' Ana Luiza Rodrigues Pellegrinelli'

Laura Augusta Barufaldi²

Wolney Lisboa Conde ${ }^{3}$

Maurício Soares Leite ${ }^{4}$

Ilaine Schuch ${ }^{5}$

Teresa Gontijo de Castro ${ }^{6}$

1 Universidade Federal de Minas Gerais, Escola de Enfermagem, Departamento de Nutrição. Belo Horizonte-MG, Brasil.

${ }^{2}$ Universidade Federal do Rio de Janeiro, Instituto de Estudos em Saúde Coletiva. Rio de Janeiro-RJ, Brasil.

${ }^{3}$ Universidade de São Paulo, Faculdade de Saúde Publica, Departamento de Nutrição. São Paulo-SP, Brasil.

${ }^{4}$ Universidade Federal de Santa Catarina, Departamento de Nutrição. Florianópolis-SC, Brasil.

${ }^{5}$ Universidade Federal do Rio Grande do Sul, Departamento de Medicina Social, Faculdade de Medicina. Porto Alegre-RS, Brasil.

${ }^{6}$ The University of Auckland, Research Fellow of the Centre for Longitudinal Research - He Ara ki MuaSchool of Population Health. Auckland, New Zealand.

Financiamento da pesquisa: Fundo de Desenvolvimento da Educação, Brasil (via Centro Colaborador em Alimentação e Nutrição do Escolar da UFRGS). Bolsa: Fundação de Amparo à Pesquisa do Estado de Minas Gerais.

Correspondência / Correspondence Teresa Gontiio de Castro Endereço eletrônico: teresagcastro108@gmail.com

\section{Resumo}

Objetivos: Descrever a distribuição de medidas bioelétricas entre os Kaingáng do Rio Grande do Sul (RS). Métodos: Estudo transversal realizado entre escolares das Terras Indígenas do RS $(\mathrm{N}=3248)$. A aferição da altura $(\mathrm{H})$ foi realizada segundo a OMS. Resistência (R) e reatância (Xc) foram obtidas por bioimpedância, sendo ajustadas por H. O ângulo de fase (AF) foi estimado pela fórmula: arc-tangente (Xc/R)*180/ח. Os valores médios (DP) das variáveis foram calculados de acordo com sexo e faixas etárias. Médias foram comparadas usando os testes t de Student e ANOVA (p <0,05). Resultados e Discussão: As proporções etárias (\%) entre os avaliados foram: crianças $(42,4)$, adolescentes $(56,0)$ e adultos/ idosos $(1,6)$. Em geral, os valores de $\mathrm{R} \mathrm{e} \mathrm{R} / \mathrm{H}$ foram maiores entre os mais jovens e no sexo feminino. Valores médios Xc e Xc/H variaram entre as faixas etárias e, em geral, valores menores de AF foram verificados entre mais jovens e do sexo feminino. Conclusões: Apesar das limitadas possibilidades de comparações, em termos gerais, as tendências de distribuição dos valores bioelétricos entre os Kaingáng confirmam achados de outras investigações. Este estudo é pioneiro na descrição de medidas bioelétricas de uma população indígena brasileira, e pretende servir de base para comparações com estudos futuros na área.

Palavras-chave: Avaliação Nutricional. Impedância Bioelétrica. Composição Corporal. Índios Sulamericanos. Saúde Indígena. 


\section{Abstract}

Describe the distribution of bioelectrical measurements among the Kaingáng from Rio Grande do Sul (RS).Cross-sectional study conducted among students of the Indigenous Lands of RS (N= 3248). Height $(\mathrm{H})$ was measured according to the OMS. Resistence $(\mathrm{R})$ and reactance $(\mathrm{Xc})$ were obtained through bioimpedance, and were adjusted by $\mathrm{H}$. The phase angle (AF) was estimated by: arc-tangente $(\mathrm{Xc} / \mathrm{R})^{*} 180 / \Pi$. Mean values (SD) and centiles of the variables were calculated by gender and age group. Means were compared using t Student and ANOVA tests $(\mathrm{p}<0.05)$. The age proportions (\%) among the evaluated were: children (42.4), adolescents (56.0) and adults/elderly (1.6). In general, values of $\mathrm{R}$ and $\mathrm{R} / \mathrm{H}$ were higher among younger individuals and females. Mean values of $\mathrm{Xc}$ and $\mathrm{Xc} / \mathrm{H}$ changed among the different age groups, and in general, lower values of AF were observed in those younger groups and females. Despite the limited possibilities of comparisons, in general way, the tendencies of distribution of the bioelectrical values among the Kaingáng are in according with results found in other investigations. This study is a pioneer in the description of bioelectrical measures for a Brazilian indigenous population, and is intended to serve as a base for comparisons with future studies in the area.

Key words: Nutritional Assessment. Bioelectrical Impedance. Body Composition. South American Indians. Indigenous Health.

\section{Introdução}

A importância de estudos que visem avaliar as condições de vida e saúde de povos indígenas no Brasil é inquestionável, dada a escassez de trabalhos desta natureza, especialmente quando se considera a imensa diversidade étnica e os dados que apontam consistentemente para indicadores de saúde amplamente desfavoráveis para este segmento da população brasileira. ${ }^{1}$

No que se refere ao estado nutricional, estudos antropométricos têm apontado prevalências importantes de déficit estatural e excesso de peso entre crianças indígenas ${ }^{2-5}$ e um gradiente de crescente excesso de peso a partir desta faixa etária. ${ }^{6-9}$ Entretanto, estudos mais detalhados do estado nutricional, empregando conjuntamente outros métodos de avaliação, são raros. A análise da bioimpedância elétrica, considerada um método rápido, não invasivo e de baixo custo ${ }^{10,11}$ foi utilizada, até o presente momento, somente em quatro estudos para avaliação da composição 
corporal entre povos indígenas no Brasil. ${ }^{12-15}$ Para isto, a partir das medidas bioelétricas, Fagundes et al, ${ }^{12}$ Lourenço et $\mathrm{al}^{13} \mathrm{e}$ Simões et $\mathrm{al}^{15}$ avaliaram a composição corporal dos indígenas do Alto Xingu/ Ikpeng, Suruí (RO) e Xucuru-Kariri (MG), respectivamente, por meio de equações preditivas. Diferentemente, o estudo de Barufaldi et a ${ }^{14}$ utilizou da Análise Vetorial de Impedância Bioelétrica (BIVA) para análise dos valores bioelétricos de Kaingáng do Rio Grande do Sul, análise esta que utiliza a própria população em estudo como referência.

No entanto, a despeito de seu reconhecido potencial para a descrição e avaliação do estado nutricional, as análises que se utilizam das medidas de bioimpedância elétrica precisam lidar com especificidades do método, e que podem constituir limitações para algumas de suas aplicações. Uma delas refere-se ao uso de equações preditivas da composição corporal em grupos populacionais distintos daqueles que deram origem às mesmas. ${ }^{11}$ De modo a superar esta limitação, diversos estudos $^{14,16-23}$ têm utilizado a Análise BIVA como alternativa às equações, uma vez que este método permite análises referenciadas na própria população em estudo, e não em outras populaçõesreferência.

O reconhecimento da importância de descrições dos parâmetros bioelétricos que compõem a análise BIVA tem, assim, resultado em esforços direcionados ao estudo das distribuições dos valores de resistência, reactância e ângulo de fase em distintas populações, e também em propostas de estabelecimento de valores de referência para valores bioelétricos. ${ }^{10,16,24,25}$ De fato, estudos verificaram que variáveis como sexo, raça/etnia, índice de massa corporal e idade influenciaram o padrão de distribuição do vetor resistência - reactância. ${ }^{10,16,24,25}$ Reforça-se, portanto, a necessidade de investigações que aprofundem o conhecimento sobre o comportamento das variáveis bioelétricas segundo estas variáveis em diferentes populações.

Neste sentido, e dada a escassez de estudos de distribuição de valores bioelétricos em populações indígenas brasileiras, o presente estudo objetivou descrever a distribuição sexo-etária dos valores de resistência $(\mathrm{R})$, reatância $(\mathrm{Xc})$ e ângulo de fase $(\mathrm{AF})$, bem como de R e Xc ajustados para altura entre indígenas matriculados nas escolas Kaingáng do Rio Grande do Sul, Brasil.

\section{Métodos}

Os Kaingáng pertencem ao tronco lingüístico Macro-Jê, e constituem um dos povos indígenas mais numerosos no Brasil. Os primeiros registros do contato com não indígenas datam ainda do século XVIII. Em 2000, a Fundação Nacional do Índio (FUNAI) reconhecia 30 Terras Indígenas desta etnia no Brasil, 12 das quais localizadas no Estado do Rio Grande do Sul (RS), o que corresponde a uma ínfima parte dos territórios tradicionalmente percorridos pela etnia. No RS 
totalizam aproximadamente 16 mil indivíduos. ${ }^{26} \mathrm{~A}$ economia tradicional Kaingáng baseava-se na caça, pesca, coleta e agricultura complementar. Atualmente a agricultura constitui elemento básico, havendo tanto roças coletivas administradas pela FUNAI como familiares. A venda de artesanato e prestação de serviços para produtores rurais também constituem fontes de renda. ${ }^{27}$ Os registros disponíveis apontam para um perfil epidemiológico caracterizado pela persistência das doenças respiratórias e infecto-parasitárias como principais causas de internação, concomitantemente ao registro crescente de obesidade e desordens associadas., ${ }^{3,28,29} \mathrm{~A}$ assistência à saúde, por sua vez, apresenta sérios problemas. ${ }^{30,31}$

Este estudo foi oriundo de um inquérito transversal de base escolar, realizado em todas as escolas indígenas localizadas em Terras Indígenas Kaingáng do Estado do Rio Grande do Sul, que estavam com situação fundiária reconhecida pela FUNAI na época do trabalho de campo, transcorrido de julho a dezembro de 2008. Em 2008 havia 5.102 estudantes indígenas matriculados nas 35 escolas das Terras Indígenas Kaingáng do Rio Grande do Sul, porém, no momento do trabalho de campo, 8,6\% deles haviam sido transferidos ou tinham se evadido das escolas, restando, efetivamente, 4662 indivíduos matriculados em julho de 2008. Destes, 896 (19,2\%) não entregaram o Termo de Consentimento Livre e Esclarecido (TCLE) assinado, 71 (1,5\%), mesmo com o TCLE assinado, se recusaram a participar da pesquisa, e, 434 (9,3\%) tiveram o TCLE assinado, mas não foram avaliados, pois estavam ausentes das escolas nos dias de avaliação.

Informações sobre sexo e idade foram coletadas diretamente dos cadastros de matrícula das escolas utilizando-se questionário estruturado. Quando a data de nascimento era inexistente nas escolas, a informação foi obtida pelo registro dos postos de saúde locais da Fundação Nacional de Saúde (FUNASA), que tinham a escola indígena sob sua adstrição (para maiores detalhes consultar Castro et al. $)^{32}$

Não foi possível obter a data de nascimento de seis indivíduos (0,13\%), apesar de estes terem sido examinados. Outros $7(0,15 \%)$ recusaram-se a participar da avaliação de impedância bioelétrica. Assim, a população do presente estudo foi constituída por 3248 indígenas Kaingáng (69,7\% dos que estavam freqüentando as escolas na época da pesquisa). Não se observaram diferenças estatisticamente significantes entre os indígenas avaliados e os não avaliados, segundo faixa etária, no tocante à proporção entre sexos. No entanto, maiores médias etárias foram observadas entre as crianças e os adolescentes não avaliados.

A mensuração dos valores de impedância bioelétrica foi realizada por meio do Impedanciômetro Quantum II ${ }^{\oplus}$ (RJL Systems, Clinton Township, Michigan, USA). Os indivíduos foram colocados em posição supina, sobre colchonete revestido com material não condutor de eletricidade e em local com temperatura ambiente. Pernas e braços foram separados, evitando-se assim contato com o 
tronco. Dois pares de eletroadesivos foram posicionados na mão e no pé do lado direito do corpo. $\mathrm{Na}$ mão, os eletroadesivos foram posicionados na linha imaginária que divide biseccionalmente a ulna e na primeira junta do dedo médio. No pé, os mesmos foram posicionados na linha imaginária que divide biseccionalmente o maléolo medial e na base do dedo médio. Os valores de resistência $(\mathrm{R})$ e reatância $(\mathrm{Xc})$ foram medidos e registrados em ohms $(\Omega)$. Considerando que a atividade física intensa pode afetar os valores de resistência, ${ }^{11,33}$ foi solicitado que nas quatro horas anteriores a esta avaliação os indivíduos não realizassem atividade física intensa. No entanto, para mensuração destes valores não foi possível o controle da temperatura ambiente, da hidratação e da realização de jejum. O ângulo de fase, em graus, foi calculado de acordo com a fórmula: arctangente (reatância/resistência)*180/ח. ${ }^{10}$ Medidas de estatura foram obtidas, em duplicata, com estadiômetro da marca AlturaExata ${ }^{\circledR}$ (Alturaexata Ltda., Belo Horizonte, Minas Gerais, Brasil), com precisão de um milímetro e capacidade de $213 \mathrm{~cm}$. A aferição desta medida foi realizada segundo recomendações da Organização Mundial da Saúde (OMS). ${ }^{34}$

A coleta de dados em campo foi realizada por quatro nutricionistas previamente capacitadas. Este estudo foi aprovado em todas as instâncias requeridas para pesquisas com povos indígenas no Brasil: Comitê de Ética em Pesquisa da Universidade Federal do Rio Grande do Sul (protocolo $n^{\circ}$. 2007726), Comissão Nacional de Ética em Pesquisa (protocolo n. 14.449), Conselho Nacional para o Desenvolvimento Científico e Tecnológico (CNPq) e Fundação Nacional do Índio (processo $n^{\circ}$. 1141/ 08 CGEP/ 08). Os dados só foram coletados de indivíduos que o autorizaram por meio da assinatura- escrita ou digital-do Termo de Consentimento Livre e Esclarecido (menores tiveram os termos assinados por seus responsáveis).

Os valores de $\mathrm{R}$ e Xc foram ajustados pela estatura $(\mathrm{R} / \mathrm{H}$ e Xc/H). Foram calculados valores médios (e respectivos intervalos com 95\% de confiança) dos parâmetros bioelétricos, de acordo com sexo e faixas etárias. Para comparações entre duas médias foi utilizado o teste $t$ de Student para amostras independentes e o teste ANOVA para comparação de três médias ou mais. As análises foram conduzidas no software SPSS 10.0, adotando-se p $<0,05$ como significativo. Todas as variáveis contínuas apresentadas neste estudo atendiam aos princípios de normalidade, de acordo com o teste de Smirnoff-Kolmogorof.

\section{Resultados}

Do total da população avaliada, 1637 (50,4\%) indivíduos pertenciam ao sexo masculino, e a idade variou entre 3 a 66,14 anos. As proporções de avaliados por faixa etária foram: 42,4\% de crianças (menores de 10 anos), 56,0\% de adolescentes (10-19 anos) e 1,6\% de adultos e idosos ( $\geq 20$ anos). 
As Tabelas 1 e 2 descrevem os valores médios e desvios-padrão de R, R/H, Xc, Xc/H e AF, respectivamente, para o sexo feminino e masculino. Para ambos os sexos, os valores médios de R e $\mathrm{R} / \mathrm{H}$ foram diferentes entre as faixas etárias, observando-se maiores valores entre indivíduos mais jovens, decrescendo com o aumento da idade. Também para ambos os sexos, os valores médios de Xc, Xc/H e AF foram estatisticamente diferentes entre as faixas etárias. Os valores médios de AF foram menores entre os indivíduos mais jovens, tanto entre homens quanto entre mulheres.

A comparação dos valores médios dos parâmetros bioelétricos entre os sexos apontou que $\mathrm{R}$ e $\mathrm{R} / \mathrm{H}$ tiveram maiores valores médios entre indígenas do sexo feminino, exceção feita para as faixas etárias de 3-6 anos, 10-11 anos, 19-20 anos e maiores de 30 anos (para R) e 3-6 anos, 10-11 anos, 11-12 anos, 12-13 anos e maiores de 30 anos (para R/H). As médias de Xc e Xc/H tiveram diferenças significativas apenas para algumas faixas etárias, especialmente entre as adolescentes de 13-17 anos, que tiveram valores médios maiores que os adolescentes (dados não apresentados em tabela). 


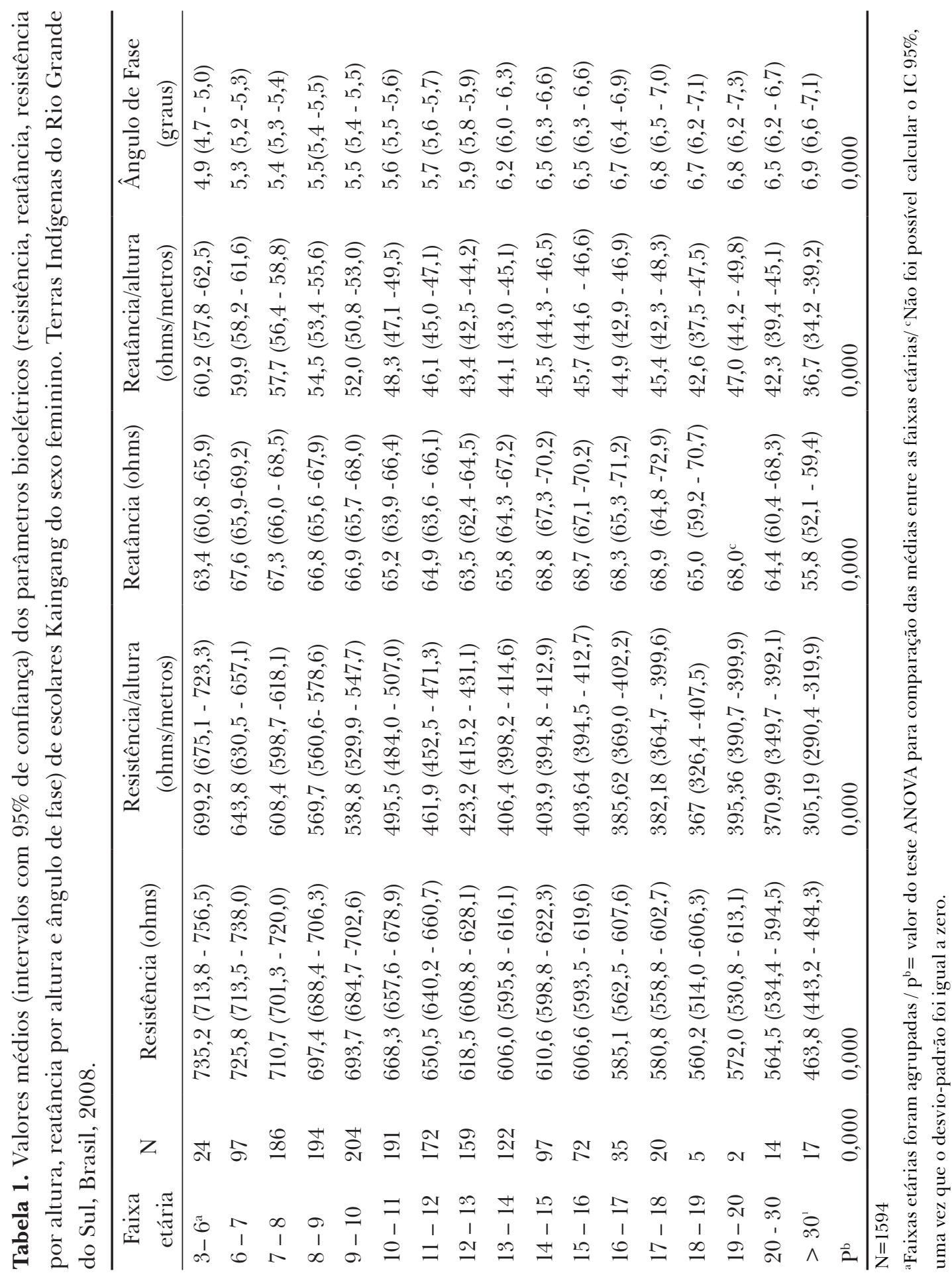




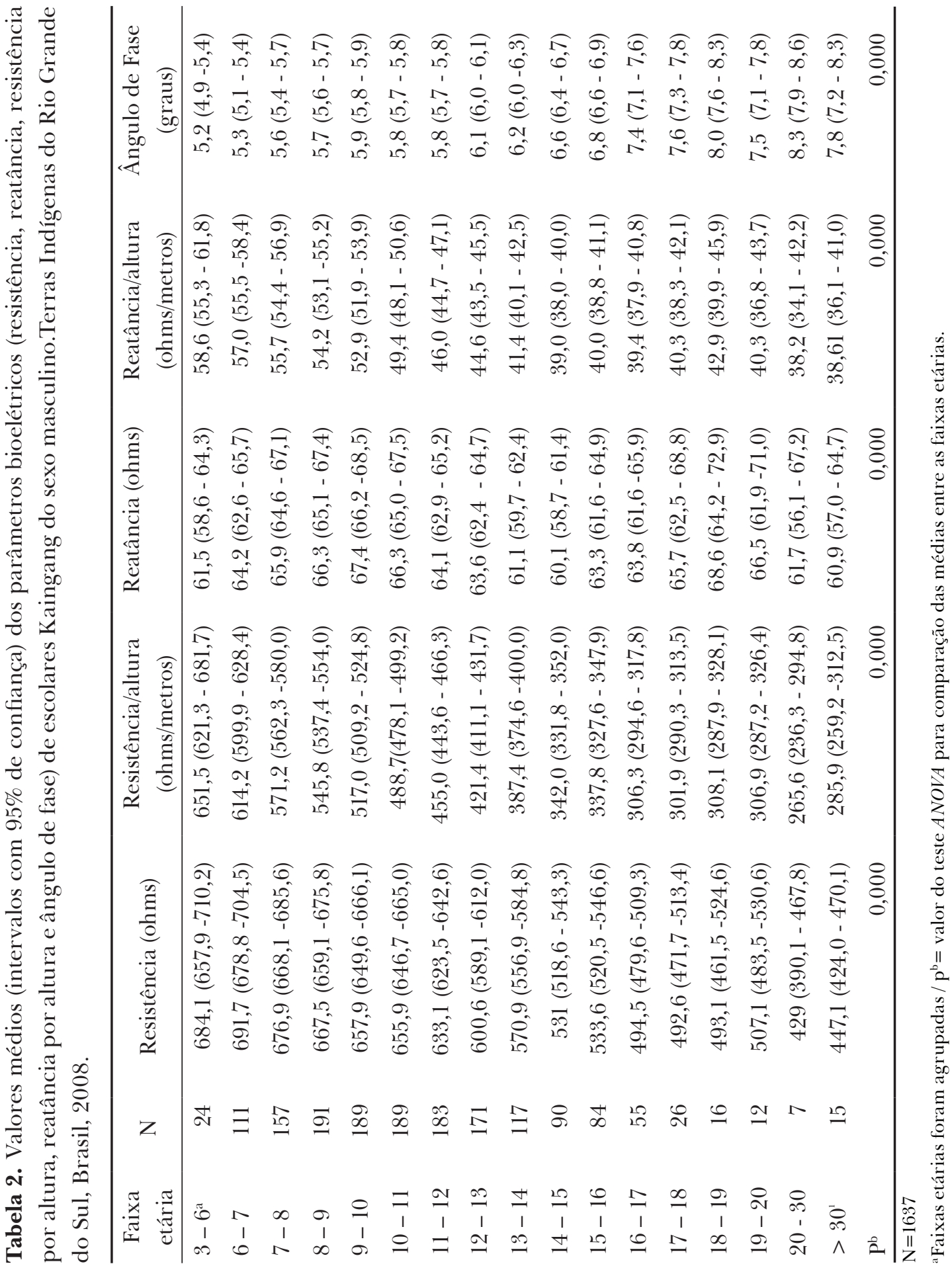




\section{Discussão}

O estudo apontou que os valores médios de resistência $(\mathrm{R})$ e resistência/altura $(\mathrm{R} / \mathrm{H})$ foram maiores entre o sexo feminino, e, para a maioria das faixas etárias, entre os mais jovens. As médias de reatância $(\mathrm{Xc})$ e $\mathrm{Xc} / \mathrm{H}$ variaram segundo as faixas etárias, e os valores médios do ângulo de fase (AF) foram, em geral, menores nos indivíduos mais jovens e maiores no sexo masculino.

A resistência é a oposição que o corpo oferece à passagem de uma corrente elétrica alternada. Em tecidos com grande quantidade de água e eletrólitos haverá melhor condução dessa corrente, gerando menores valores de resistência. Em tecidos com baixa quantidade de água, como nos tecidos ricos em lipídeos, a corrente elétrica encontrará maior dificuldade em sua condução, oferecendo assim, maiores valores de resistência. ${ }^{21}$ A reactância representa uma oposição adicional ocasionada pela capacidade de isolamento em relação à passagem da corrente elétrica por parte das membranas celulares e tecidos. Altos valores de reatância são indicativos de membrana celular saudável. ${ }^{17}$

Quando comparados os valores médios de $\mathrm{R}, \mathrm{R} / \mathrm{H}$, Xc e Xc/H deste estudo com valores do estudo de Buffa et al, ${ }^{17}$ realizado com o grupo de adolescentes italianas pré-menarca (10-14 anos) e pós-menarca (11-15 anos), estes foram semelhantes em todas as faixas de idade. O estudo de Guida et al, ${ }^{20}$ realizado com crianças italianas de oito anos, apontou valores médios de R, R/H, $\mathrm{Xc}$ e Xc/H semelhantes ao das crianças de oito anos do presente estudo. No estudo de De Palo et al, ${ }^{21}$ conduzido com indivíduos de 2-15 anos, os valores de $\mathrm{R}$ e $\mathrm{R} / \mathrm{H}$, foram sempre mais elevados que aqueles registrados entre os Kaingáng, para todas as faixas etárias. Estudo de Piccoli et al, ${ }^{16}$ realizado com 3 diferentes grupos de adultos americanos eutróficos, a partir do Terceiro Inquérito Nacional de Saúde e Nutrição -NHANES III- (não-hispânicos brancos, não-hispânicos negros e americanos-mexicanos), apontou valores médios de R, R/H, Xc e Xc/H, entre homens e mulheres na faixa etária de 20-29 anos, sempre maiores que os do presente estudo. Semelhante ao verificado entre os Kaingáng, De Palo et $\mathrm{al}^{21}$ e Piccoli et al ${ }^{16}$ encontraram maiores valores de $\mathrm{R}$ e $\mathrm{R} / \mathrm{H}$ no sexo feminino quando avaliados adolescentes italianos de 14-15 anos e adultos americanos de 20-29 anos, respectivamente.

Estudo de Maleska - Massalska et al, ${ }^{35}$ realizado entre adultos taiwaneses de 20 a 40 anos e poloneses de 20 a 26 anos encontrou valores médios de $\mathrm{R}$ e $\mathrm{R} / \mathrm{H}$ sempre inferiores aos da população Kaingáng, enquanto os achados para Xc e XC/H foram semelhantes ao registrado entre os Kaingáng do sexo masculino. O estudo de Micheli et al, ${ }^{36}$ desenvolvido entre atletas italianos do sexo masculino (20- 30 anos) encontrou valores semelhantes ao dos Kaingáng de mesmo sexo e faixa etária para $\mathrm{R} / \mathrm{H}$ e Xc/H.

Bosy - Westphal et $\mathrm{al}^{23}$ avaliaram a variação dos valores médios de $\mathrm{R}$ e Xc de acordo com o IMC de crianças, adolescentes e adultos alemães. Em geral os valores médios de R e Xc das crianças e adultos Kaingáng foram semelhantes aos valores médios apontados entre os alemães eutróficos. 
No entanto, entre os adolescentes Kaingáng de ambos os sexos de 10-13 anos os valores médios de R e Xc foram próximos aos registrados entre alemães com IMC de $20-25 \mathrm{~kg} / \mathrm{m}^{2}$, e, entre as adolescentes Kaingáng do sexo feminino entre 14-17 os valores médios de $\mathrm{R}$ se aproximaram do reportado entre as adolescentes alemães com IMC de 19 a $25 \mathrm{~kg} / \mathrm{m}^{2}$.

O ângulo de fase reflete alterações na condutividade elétrica do corpo, e indica existência ou não de alterações na integridade de membranas celulares e do espaço intercelular, refletindo, assim, a distribuição hídrica nos espaços intra e extracelulares e estando diretamente associado à quantidade de massa magra do indivíduo. Estudos sugerem que valores de ângulo de fase obtidos através da impedância bioelétrica estão relacionados aos prognósticos de morbidade e mortalidade do indivíduo. ${ }^{33}$ Os valores médios de AF encontrados no presente estudo, estratificados segundo faixa etária, foram semelhantes aos demais estudos localizados com crianças e adolescentes italianos. ${ }^{17,20,21} \mathrm{O}$ estudo de Maleska - Massalska et al, ${ }^{35}$ conduzido entre adultos, reportou menores valores de $\mathrm{AF}$ do que os registrados entre os Kaingáng.

Ressalta-se que as diferenças observadas nos padroes dos valores bioelétricos entre os diferentes sexos e faixas etárias podem estar correlacionadas em grande parte com o estado nutricional. De fato, Piccoli et $a{ }^{16}$ ilustraram que entre as mulheres existe uma maior variabilidade na estrutura dos tecidos moles e na hidratação, quando comparadas com os homens, presumivelmente devida a complexa influência hormonal. Os estudos apontaram tambem para o padrão consistente de transformação dos tecidos moles com o avanço da idade devido à perda de massa muscular a partir dos 50 anos, ${ }^{16}$ sendo a perda de tecidos moles mais acentuada no sexo masculino. ${ }^{24,37}$

O delineamento deste estudo possibilitou que se avaliasse quase $70 \%$ da população de indígenas matriculados nas escolas indígenas Kaingáng do RS. No entanto, ressalvas devem ser traçadas quanto à extrapolação dos resultados para o universo dos Kaingáng do RS e das escolas Kaingáng do estado. Primeiramente porque este estudo foi conduzido somente com indivíduos matriculados nas escolas indígenas; em segundo lugar, verificou-se uma diferença significativa na média etária entre os indivíduos que participaram do estudo e as perdas nas escolas. Ademais, os dados aqui apresentados devem ser sempre analisados levando-se em conta o fato de que neste estudo não foi possível controlar todos os aspectos influenciadores da bioimpedância, como a garantia do jejum nas 4 horas que antecederam o teste, a temperatura ambiente, o estado de hidratação, o uso de diuréticos e o estágio do ciclo menstrual (para adolescentes do sexo feminino). Adicionalmente, a interpretação destas medidas deve considerar também que, na população avaliada, havia crianças e adolescentes, indivíduos em fase de crescimento, o que pode significar, muitas vezes, valores bioelétricos temporários. ${ }^{21} \mathrm{O}$ fato de não se ter avaliado o estágio de maturação sexual dos adolescentes representa outra limitação do estudo. Buffa et a ${ }^{17}$ encontraram maiores valores médios de R/H e Xc/H entre adolescentes pré-menarca, em oposição àquelas pós-menarca. 


\section{Conclusôes}

As tendências de distribuição dos valores médios de resistência $(\mathrm{R})$, de resistência/altura $(\mathrm{R} / \mathrm{H})$ e do ângulo de fase (AF) entre os Kaingáng, segundo faixa etária e sexo, confirmam os achados de outros estudos, embora por vezes tenham variado os valores médios registrados. No entanto, em se tratando do primeiro estudo descrevendo a distribuição dos valores bioelétricos em uma população indígena, ainda são limitadas as possibilidades de comparação com outras populações desta natureza, ficando as mesmas restritas a comparações de valores registrados com grupos não indígenas. Espera-se que o presente estudo sirva de base para comparações de futuros estudos a serem realizados com indígenas, a âmbito nacional e internacional, e amplie as discussões da aliança da bioimpedância à antropometria quando da avaliação destes povos.

\section{Referências}

1. Santos, RV; Coimbra Jr CEA. Cenários e tendências da saúde e da epidemiologia dos povos indígenas no Brasil. In: Coimbra Jr. CEA, Santos RV, Escobar AL, organizadores. Epidemiologia e saúde dos povos indígenas no Brasil. Rio de Janeiro: Ed. Fiocruz, Abrasco; 2003. p.13-47.

2. Orellana JDY et al. Nutritional status and anemia in Suruí indian children, Brazilian Amazon. J. Pediatr. 2006; 85:383-8.

3. Kühl AM, Corso ACT, Leite MS, Bastos JL. Perfil nutricional e fatores associados à ocorrência de desnutrição entre crianças indígenas Kaingang da Terra Indígena de Mangueirinha, Paraná, Brasil. Cad. Saude Publica 2009;25(2):409-20.

4. Leite MS, Leite MS, Santos RV, Coimbra Jr CEA, Gugelmin SA, Kac G, et al. Alimentação e nutrição dos povos Indígenas no Brasil. In: Kac G, Sichieri R, Gigante DP, organizadores. Epidemiologia nutricional. Rio de Janeiro: Editora Fiocruz, Atheneu; 2007. p. 503-18.

5. Horta BL et al. Nutritional status of indigenous children: findings from the Firsty National Survey of Indigenous People's Health and Nutrition in Brazil. Int. J. Equity Health 2013; 12 (23):1-13.

6. Cardoso AM et al. Inquérito nacional de saúde e nutrição dos povos indígenas. Rio de Janeiro: FUNASA, ABRASCO, Banco Mundial, 2009.

7. Leite MS, Santos RV, Gugelmin AS, Coimbra Jr CEA. Crescimento físico e perfil nutricional da população indígena Xavánte de Sangradouro-Volta Grande, Mato Grosso, Brasil. Cad. Saúde Publica 2006; $22(2): 265-76$.

8. Gugelmin AS, Santos RV. Uso do Índice de Massa Corporal na avaliação do estado nutricional de adultos indígenas Xavánte, Terra Indígena Sangradouro-Volta Grande, Mato Grosso, Brasil. Cad. Saúde Publica 2006; 22(9):1865-72.

9. Gimeno AS, Rodrigues D, Canó EN, Lima EE, Schaper M, Pagliaro H, et al. Cardiovascular risk factors among Brazilian Karib indigenous peoples: Upper Xingu, Central Brazil, 2000. J. Epidemiol. Comm. Health 2009; 63(4):299-304. 
10. Barbosa-Silva MCG, Barros AJD, Wang J, Heymsfield SB, Pierson Jr RN, et al. Bioelectrical impedance analysis: population reference values for phase angle by age and sex. Am. J. Clin. Nutr. 2005; 82(1):49-52.

11. Dehghan M, Merchant AT. Is bioelectrical impedance accurate for use in large epidemiological studies? Nutr. J. 2008; 7:1-7.

12. Fagundes U, Kopelman B, oliva CAG, Baruzzi RG, Fagundes Neto U. Avaliação do estado nutricional e da composição corporal das crianças índias do Alto Xingu e da etnia Ikpeng. J. Pediatr. 2004; 80(6):483-489.

13. Lourenço AEP, Santos RV, Orellana JDY, Coimbra Jr CEA. Nutrition transition in Amazonia: obesity and socioeconomic change in the Suruí Indians from Brazil. Am. J. Hum. Biol. 2008; 20:564-571.

14. Barufaldi LA et al. Bioelectrical Impedance values among indigenous children and adolescents in Rio Grande do Sul, Brazil. Rev. Panam. Salud Publica 2011; 30(1):39-45.

15. Simões BS, Machado-Coelho GLL, Pena JL, Freitas SN. Perfil nutricional dos indígenas XukuruKariri, Minas Gerais, de acordo com diferentes indicadores antropométricos e de composição corporal. Ciênc. Saúde Coletiva 2013; 18 (2):405-411.

16. Piccoli A, Pillon L, Dumler F. Impedance vector distribution by sex, race, body mass index, and age in the United States: standard reference intervals as bivariate z scores. Nutrition 2002; 18(2):153-167.

17. Buffa R, Floris G, Marini E. Bioelectrical impedance vector in pre- and postmenarcheal females. Nutrition 2002; 18(6):474-478.

18. Buffa R, Floris G, Marini E. Assessment of nutritional status in free-living elderly individuals by bioelectrical impedance vector analysis. Nutrition 2009; 25(1):3-5.

19. Buffa R, Baali A, Lahmam Um, Amor H, Zouini M, Floris G, et al. Assessment of nutritional status in the Amazigh children of Amizmiz (Azgour Valley, High Atlas and Morocco). J. Trop. Pediatr. 2009; 55(6):406-408.

20. Guida B, Pietrobelli A, Trio R, Laccetti R, Falconi C, Perrino NR, et al. Body mass index and bioelectrical vector distribution in 8-year-old children. Nutr. Metab. Cardiovasc. Dis. 2008; 18(2):133-141.

21. De Palo T, Messina G, Edefonti A, Perfumo F, Pisanello L, Peruzzi L, et al. Normal Values of the Bioelectrical Impedance Vector in Childhood and Puberty. Nutrition 2000; 16(6):417-424.

22. Savino F, Grasso G, Cresi F, Oggero R, Silvestro L. Bioelectrical impedance vector distribution in the first year of life. Nutrition 2003; 19(6):492-6.

23. Bosy-Westphal A, Danielzik S, Dörhöfer RP, Piccoli A, Müller MJ. Patterns of bioelectrical impedance vector distribution by body mass index and age: implications for body-composition analysis. Am. J. Clin. Nutr 2005; 82(1):60-8.

24. Saragat B, Buffa R, Mereu E, De Rui M, Coi A, Sergi G, et al. Specific bioelectrical impedance vector reference values for assessing body composition in the Italian elderly. Exp. Gerontol. 2014; 50:52-6.

25. Margutti AV, Monteiro JP, Camelo JS Jr. Reference distribution of the bioelectrical impedance vector in healthy term newborns. Br. J. Nutr. 2010; 104(10):1508-13. 
26. Ricardo CA. organizador. Povos indígenas no Brasil 1996/2000. São Paulo: Instituto Socioambiental; 2000.

27. Veiga J. Aspectos fundamentais da cultura Kaingáng. São Paulo: Curt Nimuendajú; 2006. 254 p.

28. Menegolla IA, Drachler ML, Rodrigues IH, Schwingel LR, Scapinello E, Pedroso MB, et al. Estado nutricional e fatores associados à estatura de crianças da Terra Indígena Guarita, Sul do Brasil. Cad. Saúde Pública 2006; 22:395-406.

29. Gilio J, Mioranza SL, Takizawa MGMH. Parasitismo intestinal em índios da reserva indígena de Rio das Cobras. Rev. Bras. Anal. Clin. 2006; 38:193-5.

30. Hökerberg YHM, Duchiade MP, Barcellos C. Organização e qualidade da assistência à saúde dos índios Kaingang do Rio Grande do Sul. Cad. Saúde Publica 2001;17(2):261-72.

31. Diehl EE. Agravos na saúde Kaingang (Terra Indígena Xapecó, Santa Catarina) e a estrutura dos serviços de atenção biomédica. Cad. Saúde Pública 2001;17(2):439-45.

32. Castro TG, Schch I, Conde WL, Veiga J, Leite MS, Dutra CLC, et al. Estado Nutricional dos indígenas Kaingang matriculados em escolas indígenas do estado do Rio Grande do Sul, Brasil. Cad. Saúde Publica 2010; 26(9):1766-76.

33. Baumgartner RN, Electrical impedance and total body electrical conductivity. In: Roche AF, Heymsfield S, Lohman TG. Human body composition. Champaing, IL: Human Kinetics; 1996. p. 79-107.

34. World Health Organization. Physical status: the use and interpretation of anthropometry. Geneva: WHO; 1995. Technical Report Series 854.

35. Maleska-Massalska T Smolen A, Madro E, Surtel W. Bioimpedance vector pattern in Taiwanese and Polish college students detected by bioelectric impedance vectorial analysis: preliminary observations. The Scientific World J [Internet]. 2012; 1-5. Disponível em: http://www.researchgate. net/publication/224972728_Bioimpedance_Vector_Pattern_in_Taiwanese_and_Polish_College_ Students_Detected_by_Bioelectric_Impedance_Vector_Analysis_Preliminary_Observations

36. Micheli ML, Pagani L, Marella M, Gulisano M, Piccoli A, Angelini F, et al. Bioimpedance and impedance vector patterns as predictors of male elite soccer players. Int. J. Sports Physiol. Perform. 2014; 9(3):532-539.

37. Guida B, Laccetti R, Gerardi C, Trio R, Perrino NR, Strazzullo P, et al. Bioelectrical impedance analysis and age-related differences of body composition in the elderly. Nutr. Metab. Cardiovasc. Dis. 2007; 17(3):175-180. 
